Supporting Information

“Plug and Play" Functionalized Erythrocyte Nanoplatform for Target

\title{
Atherosclerosis Management
}

Yuan Zhong, ${ }^{\dagger}$ Xian Qin, ${ }^{\dagger}$ Yi Wang, Kai Qu, Li Luo, Kun Zhang, Boyan Liu, Essam Abdo Mohammed Saad Obaid, Wei Wu,* and Guixue Wang*

Key Laboratory for Biorheological Science and Technology of Ministry of Education, State and Local Joint Engineering Laboratory for Vascular Implants, Bioengineering College of Chongqing University, Chongqing, 400030, China

*Email: david2015@cqu.edu.cn.

*Email:wanggx@cqu.edu.cn.

† Y.Z. and X.Q. contributed equally to this work. 


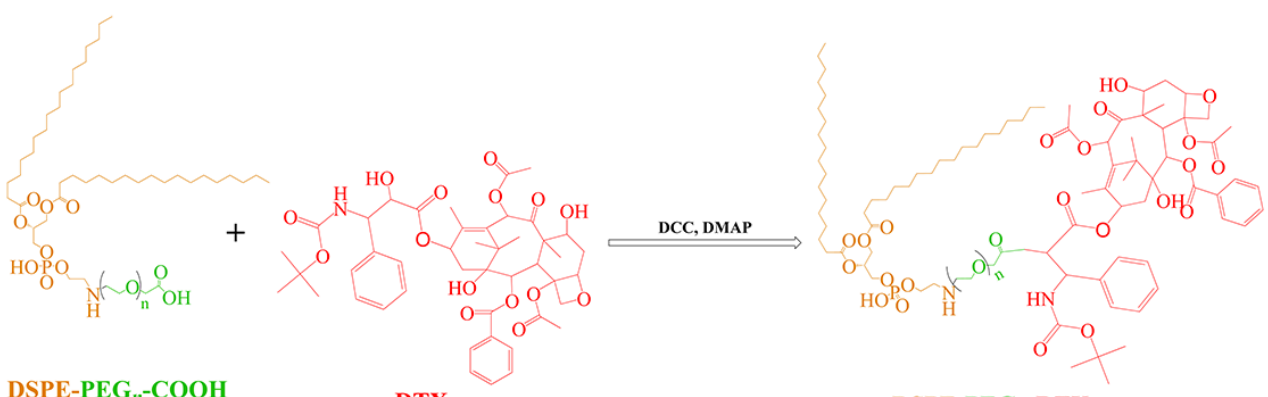

DSPE-PEG ${ }_{n}-\mathrm{COOH}$

DTX

DSPE-PEG $_{n}$-DTX
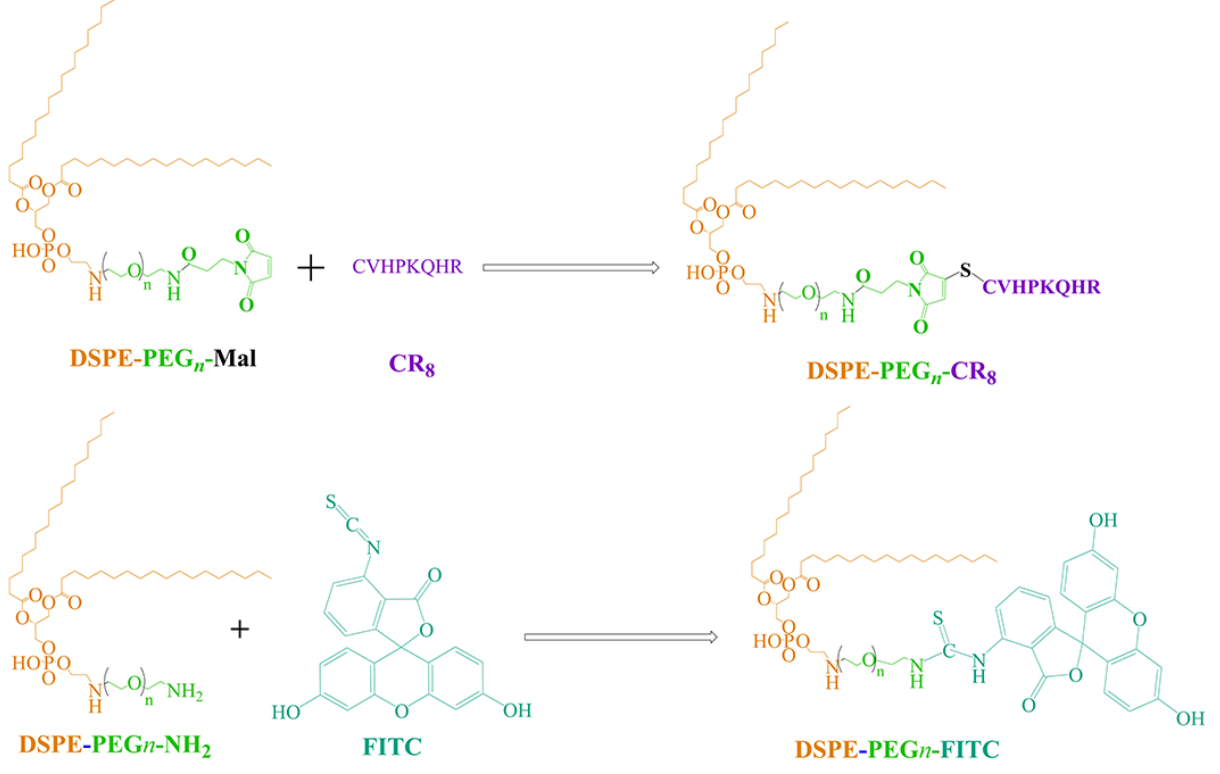

Figure S1. Synthetic route of DSPE-PEG $2000-\mathrm{DTX}, \mathrm{DSPE} \mathrm{PEG}_{2000}-\mathrm{CR}_{8}$ and DSPE-PEG $2000-\mathrm{FITC}$.

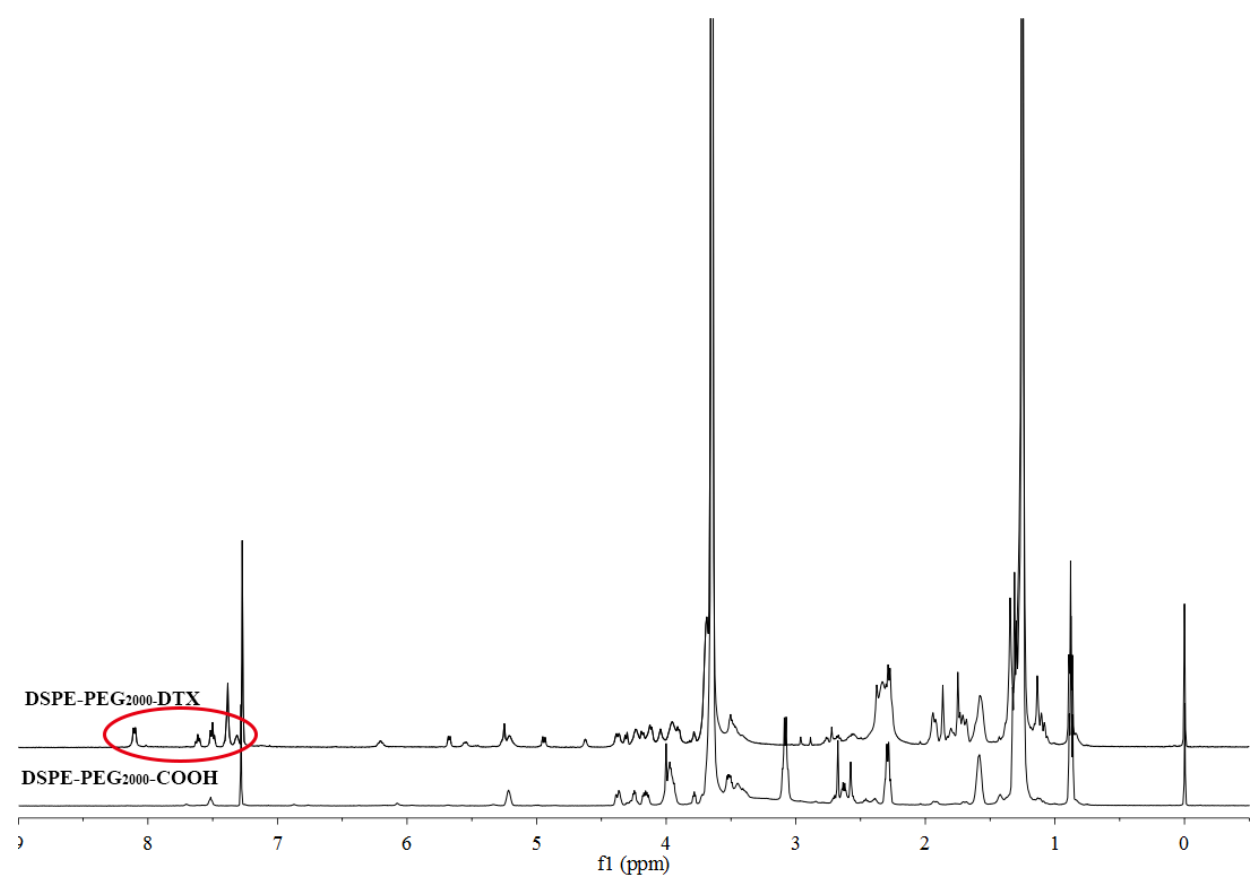

Figure S2. ${ }^{1} \mathrm{H}$ NMR spectrum of DSPE-PEG $2000-\mathrm{DTX}$. 


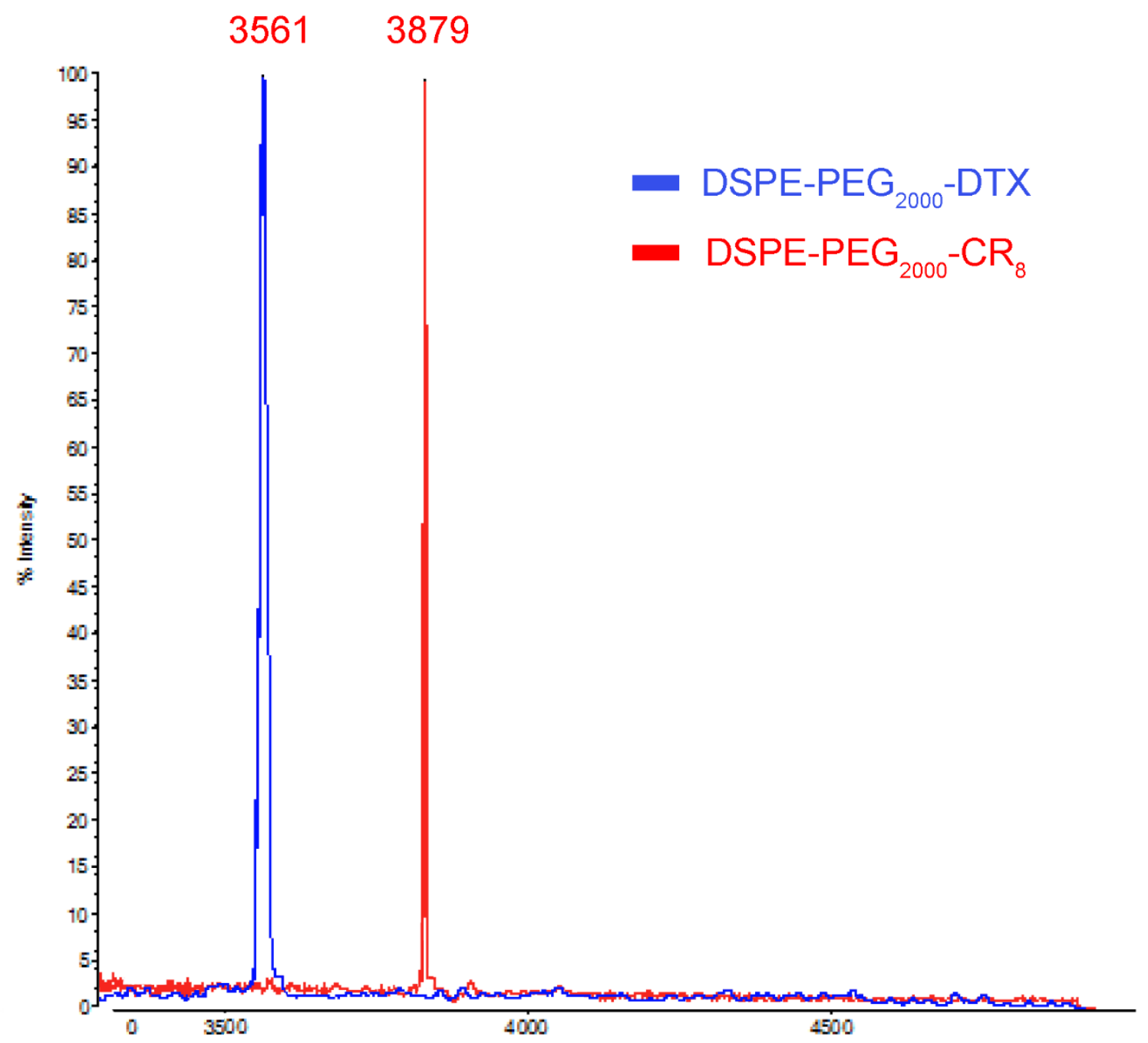

Figure S3. MALDI-TOF-MS spectra of DSPE-PEG $2000-\mathrm{DTX}$ and DSPE-PEG ${ }_{2000}-\mathrm{CR}_{8}$. 


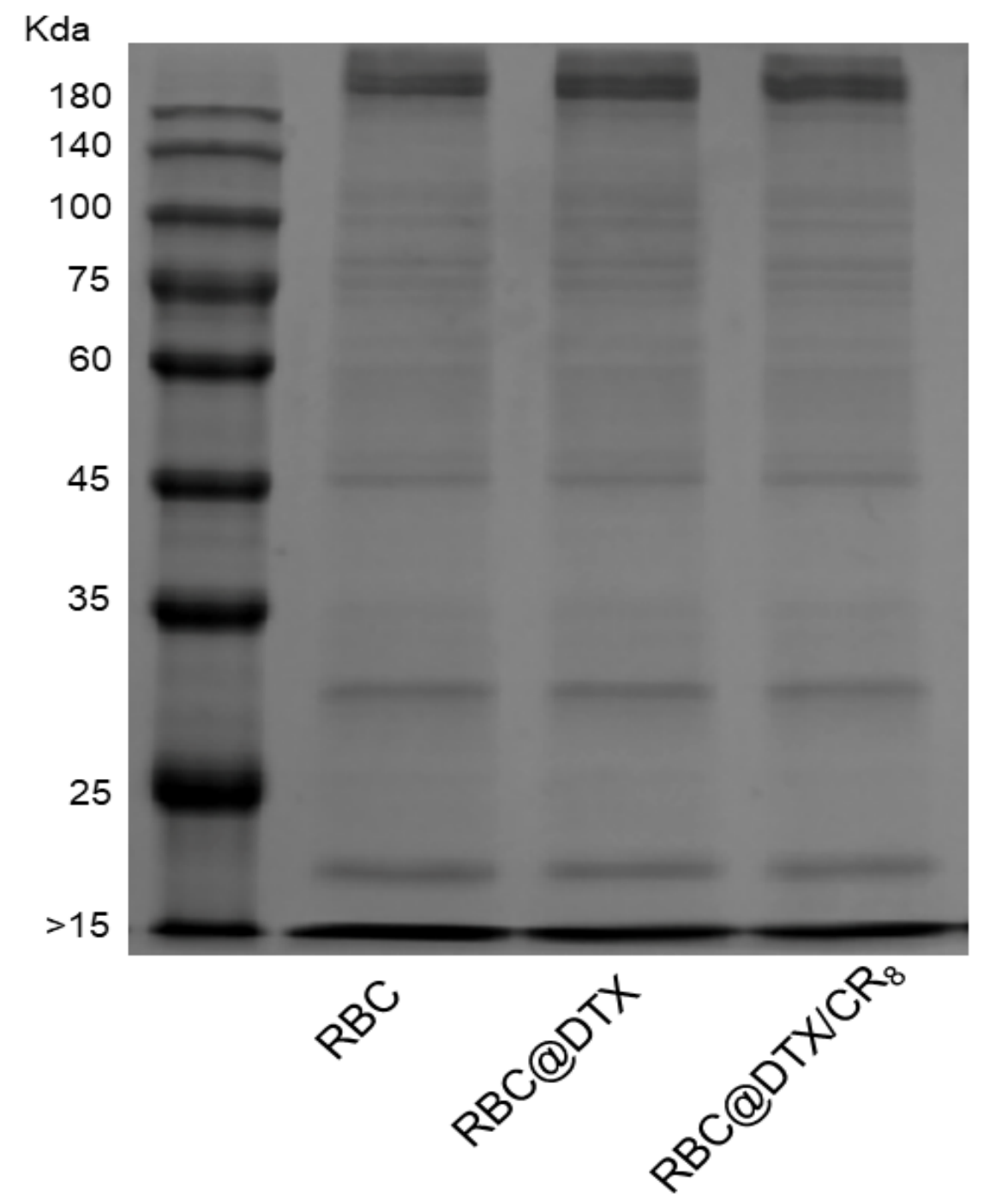

Figure S4. SDS-PAGE of RBC, RBC@DTX and RBC@DTX/CR8.

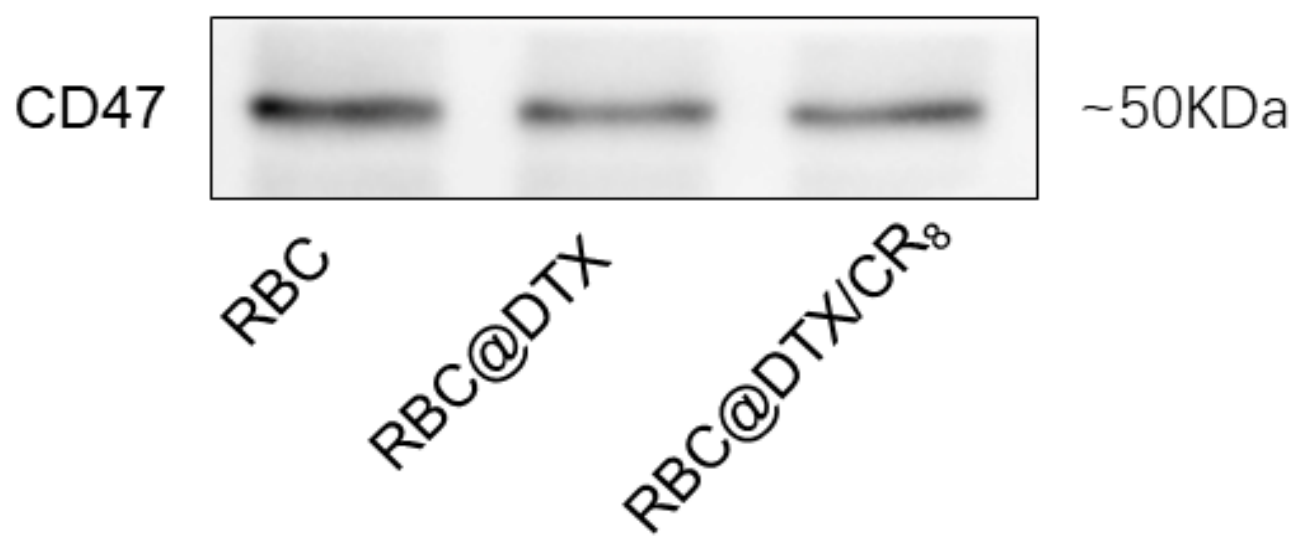

Figure S5. Western blotting of RBC, RBC@DTX and RBC@DTX/CR 8 . 

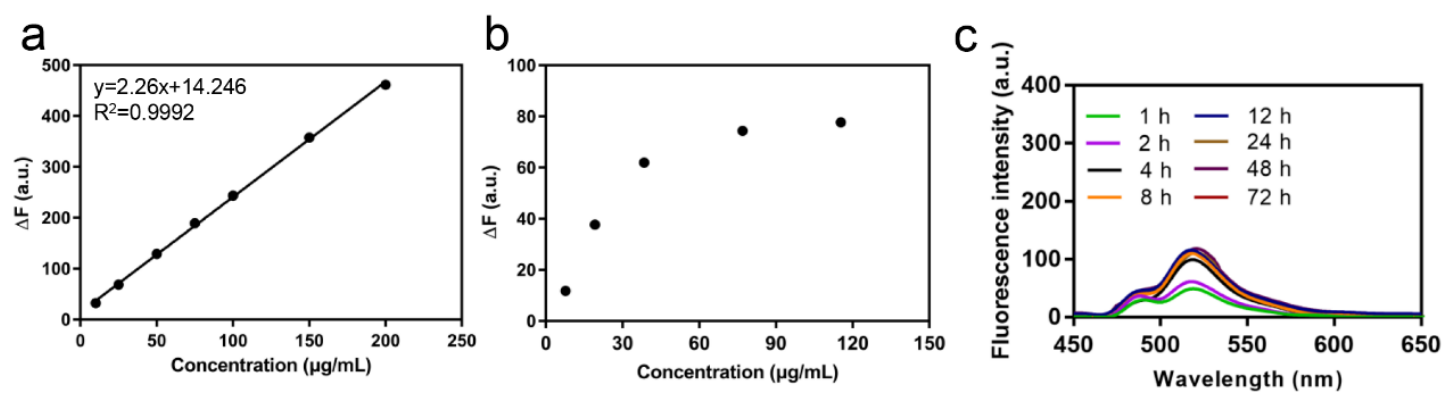

Figure S6. (a) Standard curve of fluorescence intensity in different concentration of DSPE-PEG $2000-$ FITC. (b) The relationship between co-incubation concentration and fluorescence intensity. (c) The fluorescence intensity of DSPE-PEG ${ }_{2000}$-FITC in dialysate.
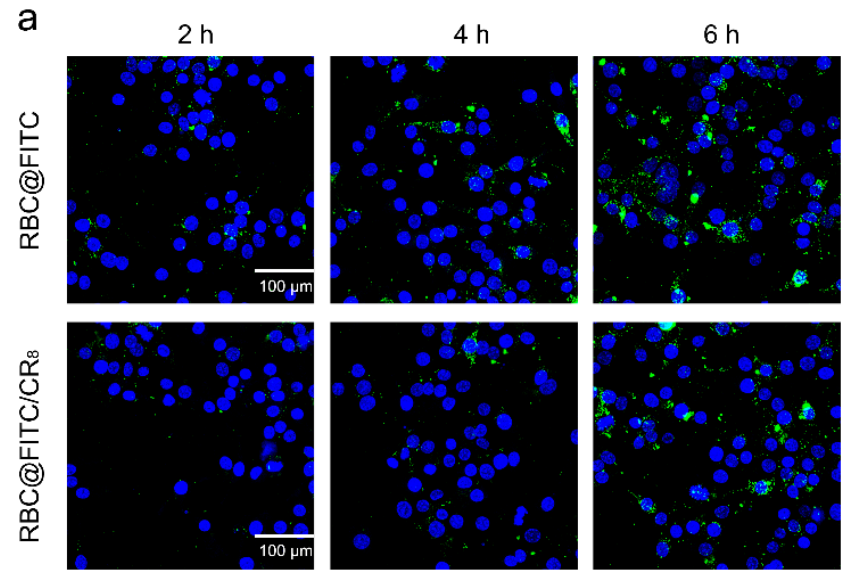

$\mathrm{b}$
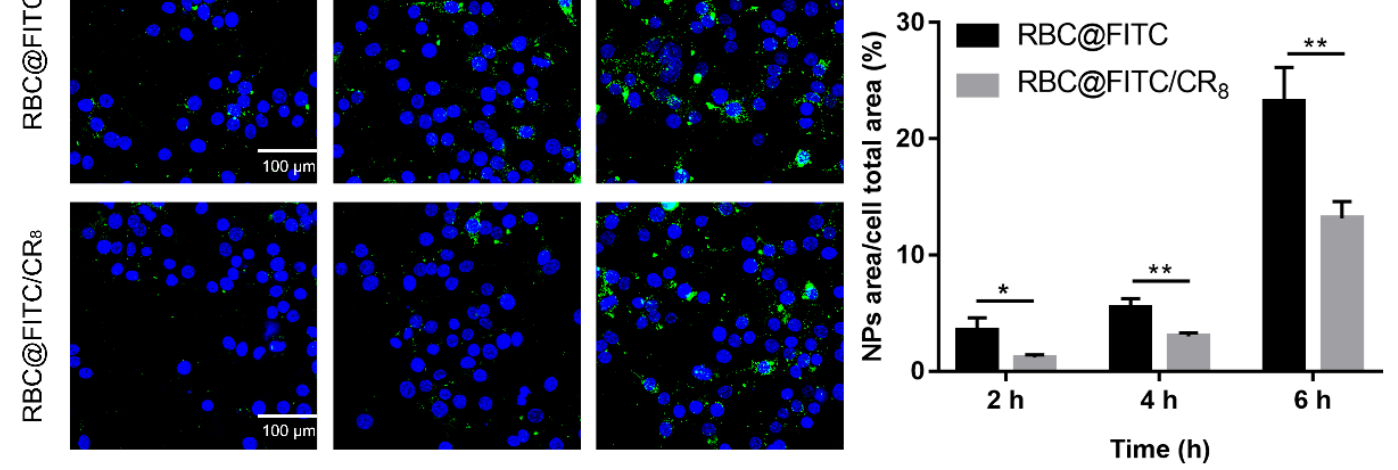

Figure S7. (a) CLSM images of RBC@FITC and RBC@FITC/CR8 endocytosed by RAW 264.7. The blue is the nucleus stained by DAPI, the green is the nano-platforms. The scale bar is $100 \mu \mathrm{m}$. (b) The ratio of green fluorescence to cell total area. The difference significance levels were set at $* p$ $<0.05, * * p<0.01, * * * p<0.001$, and $* * * * p<0.0001$. 
a

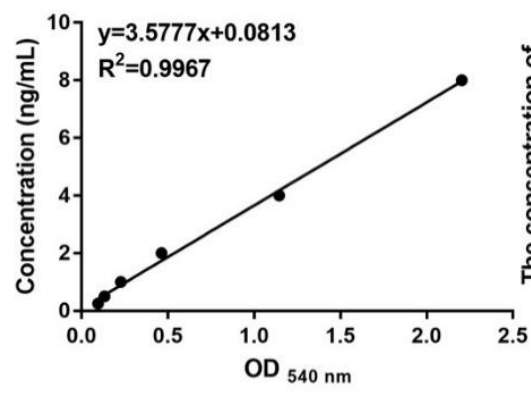

b

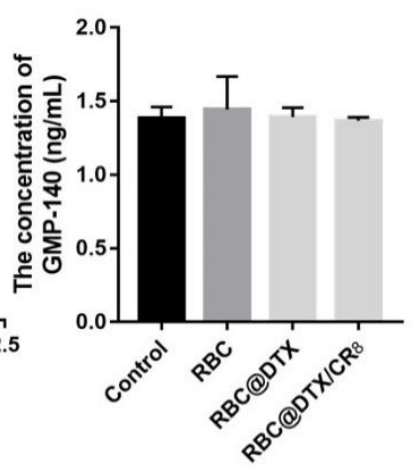

Figure S8. (a) The standard curve of the $\alpha$-granule membrane protein (GMP-140), (b) The concentration of GMP-140 in plasma after incubation with different samples $(n=3)$.

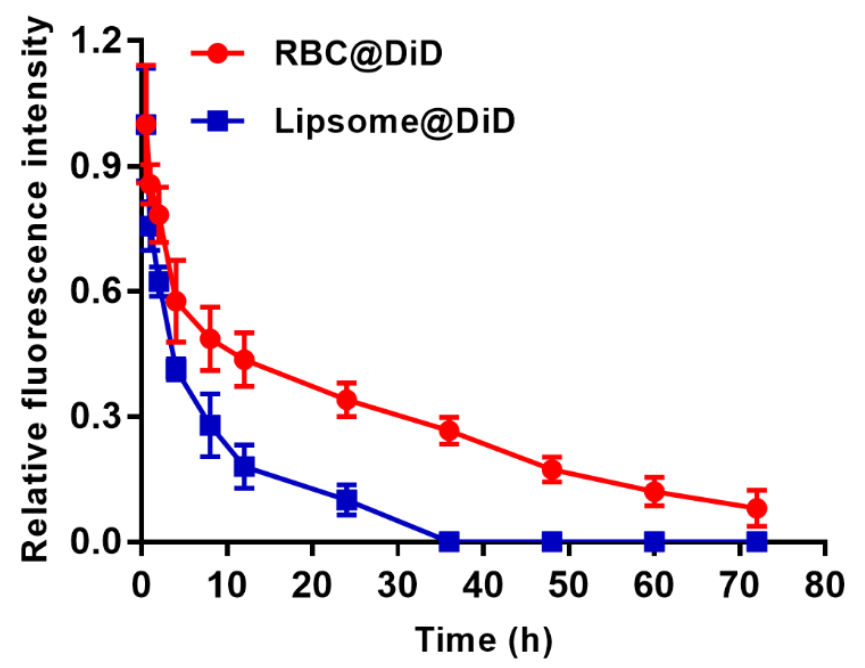

Figure S9. Relative fluorescence intensity of RBC@DiD and Liposome@ DiD for pharmacokinetic studies in C57BL/6 mice $(n=3)$. 


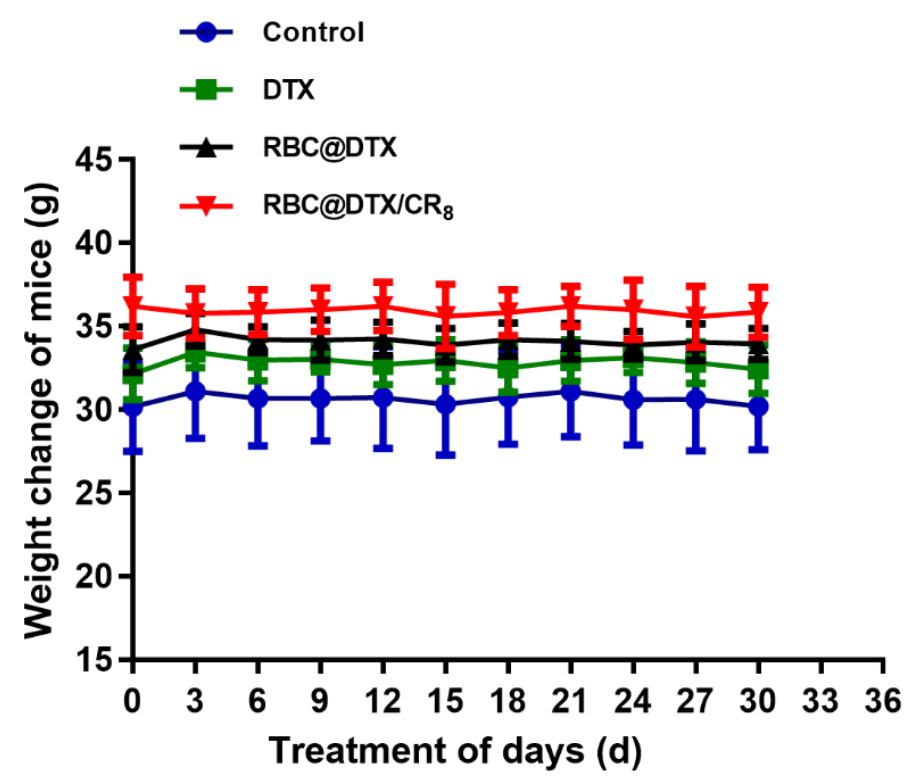

Figure S10. Weight changes of mice during the treatment.

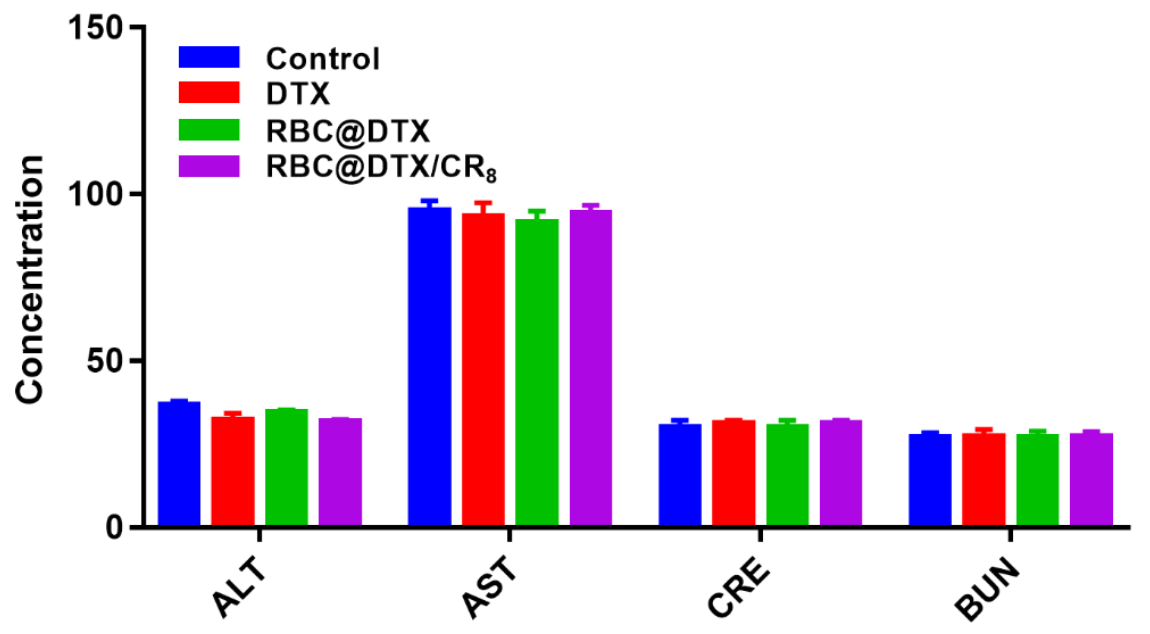

Figure S11. The blood biochemical assays of alanine aminotransferase (ALT), aspartate aminotransferase (AST), creatinine (CRE), and blood urea nitrogen (BUN). 


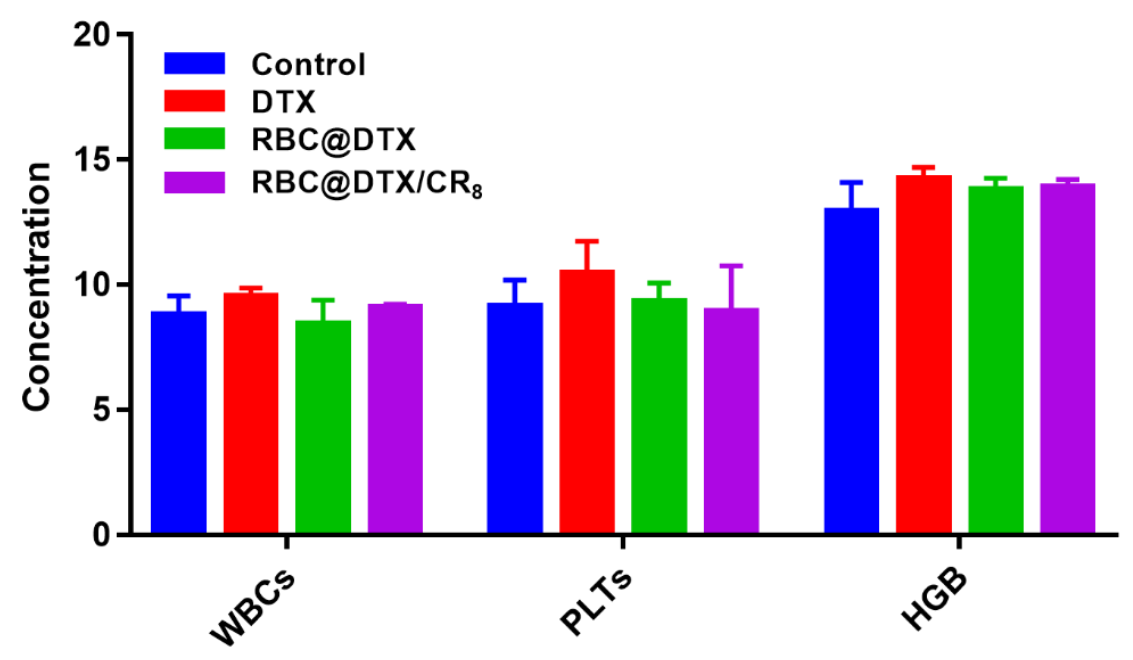

Figure S12. The routine blood examination of RBCs, white blood cells (WBCs), platelets (PLTs), and hemoglobin (HGB).

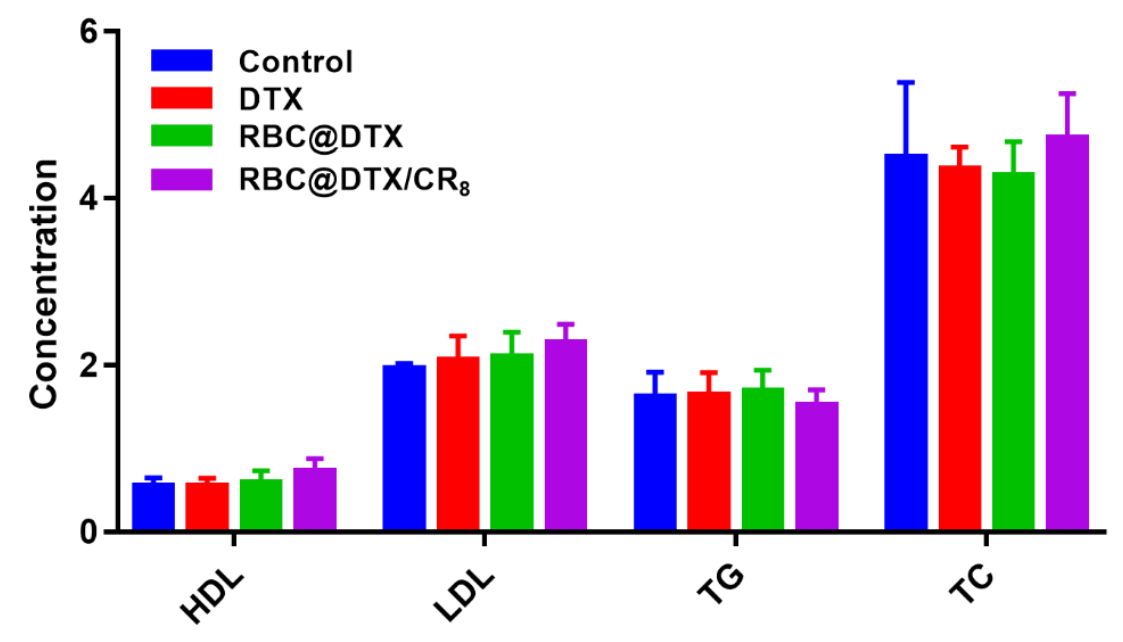

Figure S13. The blood lipid levels of highly density lipoprotein (HDL), low density lipoprotein (LDL), triglyceride (TG), and total cholesterol (TC).

Table S1. Summary of the hemolysis ratio caused by DTX, RBC@DTX, RBC@DTX/CR 8 .

\begin{tabular}{lccccc}
\hline \multicolumn{5}{c}{ Hemolysis ratio $(\%)$} \\
\hline Sample $(\boldsymbol{\mu g} / \mathbf{m L})$ & $\mathbf{1 0}$ & $\mathbf{2 0}$ & $\mathbf{3 0}$ & $\mathbf{4 0}$ & $\mathbf{5 0}$ \\
DTX & $0.09 \pm 0.01$ & $0.82 \pm 0.02$ & $0.33 \pm 0.04$ & $0.58 \pm 0.01$ & $0.07 \pm 0.02$ \\
RBC@DTX & $3.25 \pm 0.18$ & $2.09 \pm 0.03$ & $0.60 \pm 0.24$ & $2.18 \pm 0.14$ & $3.66 \pm 0.05$ \\
RBC@DTX/CR 8 & $2.72 \pm 0.16$ & $2.80 \pm 0.08$ & $4.37 \pm 0.28$ & $3.15 \pm 0.04$ & $3.16 \pm 0.10$ \\
\hline
\end{tabular}

